\title{
Babylonian and Greek Astronomy in a Papyrus Concerning Mars
}

by

\author{
ALEXANDER JONES*
}

In 1960 Neugebauer established that two Greco-Egyptian papyrus fragments, $P$. Heid. Inv. 4144 and the already published $P$. Mich. 151 , once belonged to a single set of (at least) six numerical tables, dated on palaeographical grounds to the third century of our era. ${ }^{1}$ Since the names of the zodiacal signs Taurus and Gemini appear at the head of the last preserved table, the papyrus must have had something to do with astronomy or astrology. In his original publication Neugebauer discussed the simple arithmetical structure of the tables, but he was unable to explain their purpose. Later, he realized that they were related to the Babylonian System A scheme for computing the phases of Mars, which is based on a division of the ecliptic into six zones coinciding with consecutive pairs of zodiacal signs. ${ }^{2}$ Like the Babylonian scheme, the papyrus associated with each of the six zones a constant used to determine the planet's progress from one synodic period to the next; but the constants in the papyrus were not the same as their counterparts in the Babylonian theory. Neugebauer concluded that the papyrus "seems to be clear evidence for the existence of a greatly modified Babylonoid theory of Mars although we can only guess at its working."

With even this incomplete understanding of the papyrus, we can see that it is significant for the history of the poorly documented Greek mathematical astronomy that Ptolemy's Almagest superseded. Since the beginning of this century we have known that some elements of Babylonian mathematical astronomy were known to

\footnotetext{
* Institute for the History and Philosophy of Science and Technology, University of Toronto, Toronto M5S 1K7, Canada.
} 
Greek astronomers (in particular Hipparchus, in the latter half of the second century B.C.); ${ }^{3}$ but how much was transmitted, and to what degree the development of Greek astronomy was affected by this transmission, have been disputed questions. Neugebauer, for example, was inclined to believe that little more than a few concepts, numerical parameters, and simple procedures passed from the Babylonians to the Greeks. ${ }^{4}$ On the other hand van der Waerden has attempted in a series of articles to demonstrate that Greco-Egyptian astronomical Almanacs (texts giving the dates during a series of years when the planets entered each zodiacal sign) were at least partly computed according to Babylonian methods. ${ }^{5}$ Neugebauer expressed doubts about the cogency of these analyses, ${ }^{6}$ but van der Waerden's argument that the data for Mars in one Almanac (the "Stobart Tablets", covering A.D. 70 to 133) were based on the Babylonian System A scheme has withstood the most sceptical scrutiny. Now we actually have part of a Greek scheme that used the System A zones for Mars. Taking into account the fragmentary nature of our evidence, we are entitled to infer that what is well documented for one planet was probably true for them all: that is, Babylonian predictive schemes for all the planets were transmitted to the Hellenistic world, and continued to be used as late as a century after Ptolemy. ${ }^{7}$.

But it is also noteworthy that the papyrus tables attest to changes in the Babylonian scheme. Van der Waerden's analysis of the Mars data in the Stobart Tablets showed that they were computed not only using the original six Babylonian zones, but also using the Babylonian constants associated with the zones. There must have been some reason why an unknown astronomer threw these constants out, and put new ones in their stead. The principal purpose of this article is to show that the purpose of this modification was to bring the Babylonian arithmetical scheme into agreement with a Greek geometrical model of Mars's motion.

The object of the Babylonian System A scheme was to predict the longitudes and dates of the cycle of Mars's phases, i.e. first and last visibility, the two stationary points, and the 'acronychal rising' (rising at sunset, near opposition). ${ }^{8}$ The scheme's basic structure consists of (1) finding the longitudes of a succession of certain phases (last visibility, first visibility, first station) from their longitudes in 
an initial synodic cycle, (2) finding the corresponding dates of these phases, and (3) finding the longitude and date of the acronychal rising and second station in each cycle from the longitude and date of the immediately preceding first station. We need only concern ourselves with step (1). The synodic arc, i.e. the planet's progress in longitude from one occurrence of a particular phase to the next occurrence of the same phase, is determined as a function of the 'starting longitude', or longitude of the planet at the first occurrence. The scheme divides the ecliptic into six equal zones, and associates a constant 'ideal synodic arc' $w_{i}$ with each zone $i$ :

$\begin{array}{ccc}i & \text { zone } & \text { synodic arc }\left(w_{i}\right) \\ 1 & 00^{\circ}-\pi 30^{\circ} & 45 \\ 2 & 00^{\circ}-\Omega 30^{\circ} & 30^{\circ} \\ 3 & m 0^{\circ}-\simeq 30^{\circ} & 40^{\circ} \\ 4 & m 0^{\circ}-\approx 30^{\circ} & 60^{\circ} \\ 5 & 60^{\circ}-=30^{\circ} & 90^{\circ} \\ 6 & \times 0^{\circ}-r 30^{\circ} & 67 ; 30^{\circ}\end{array}$

If a starting longitude $\lambda_{0}$ falls in zone $i$, and the number of degrees $r$ between $\lambda_{0}$ and the beginning of zone $i+1$ is greater than $w_{i}$, then the synodic arc $\Delta \lambda$ is simply $w_{i}$. Otherwise one uses the following interpolation rule:

$$
\Delta \lambda=w_{i+1}-\frac{w_{i+1}-w_{i}}{w_{i}} \cdot r
$$

The values of $w_{i}$ are such that the 'transition coefficient' $\frac{w_{i+1}-w_{i}}{w_{i}}$ is always a simple sexagesimal fraction, so that the longitudes of a succession of phases of one kind can be computed by easy arithmetic.

The surviving cuneiform texts say nothing about how these rules evolved. Recently Aaboe has proposed a convincing and surprisingly simple reconstruction of the steps by which the zone patterns in schemes of the System A type must have been derived from observations. ${ }^{9}$ The only prerequisites are a continuous record 
of observations of a planet's phases (perhaps giving no more than the date and zodiacal sign) extending over several decades, and a reasonably good period relation between the planet's synodic and longitudinal periods. These requirements are fulfilled by the Babylonian astronomical Diaries, which preserved a (probably) continuous record of observations from the seventh century B.C. on, and period relations such as the "Goal Year" periods. ${ }^{10}$ The 'model' consists of very straightforward assumptions concerning the way that incidences of the planet's phases are unequally distributed over the ecliptic; the parameters of the model are found simply by counting all the occurrences of the phases observed in arbitrarily chosen zones of the ecliptic during a period containing approximately a whole number of years and a whole number of synodic cycles. The $w$ value associated with each zone is easily determined from the tally of phases counted in it, although some tinkering with the w's or with the zone boundaries would usually be necessary to obtain convenient transition constants and to eliminate long term deviations.

How good are the predictions of the System A Mars scheme? Aaboe plotted the synodic arc predicted by System A as a function of the starting longitude (taking the transitions into account), and found very good agreement with the actual synodic arcs resulting from an approximate simulation of Keplerian motion. ${ }^{11}$ The errors, moreover, tend to cancel each other out even in the short term, while the precision of the long period relations assumed in all the Babylonian planetary schemes prevents long-term deviations. Comparing a series of eight consecutive first stations of Mars computed according to System A and modern theory, I found discrepancies in longitude never exceeding $3 \frac{1}{2}^{\circ}$. It seems that the theory's predictions are well within the tolerance that Babylonian astronomers would have allowed for their observations.

Now let us see how the System A scheme is modified in our Greek papyrus. The two fragments $P$. Heid. Inv. 4144 and P. Mich. 151 are the remains of a typical papyrus scroll of approximately $25 \mathrm{~cm}$, or 45 lines, height. The original situation of the fragments is shown in Fig. 1. ${ }^{12}$ Parts of six successive numerical tables (here numbered $i$ to vi) are preserved; what preceded and followed them is entirely open to conjecture. The tables all followed a uniform arithmetical pattern, which makes it possible to reconstruct tables ii through vi 
$\begin{array}{lllll}\text { A } & \text { B } & \text { C } & \text { D } & {[E]}\end{array}$

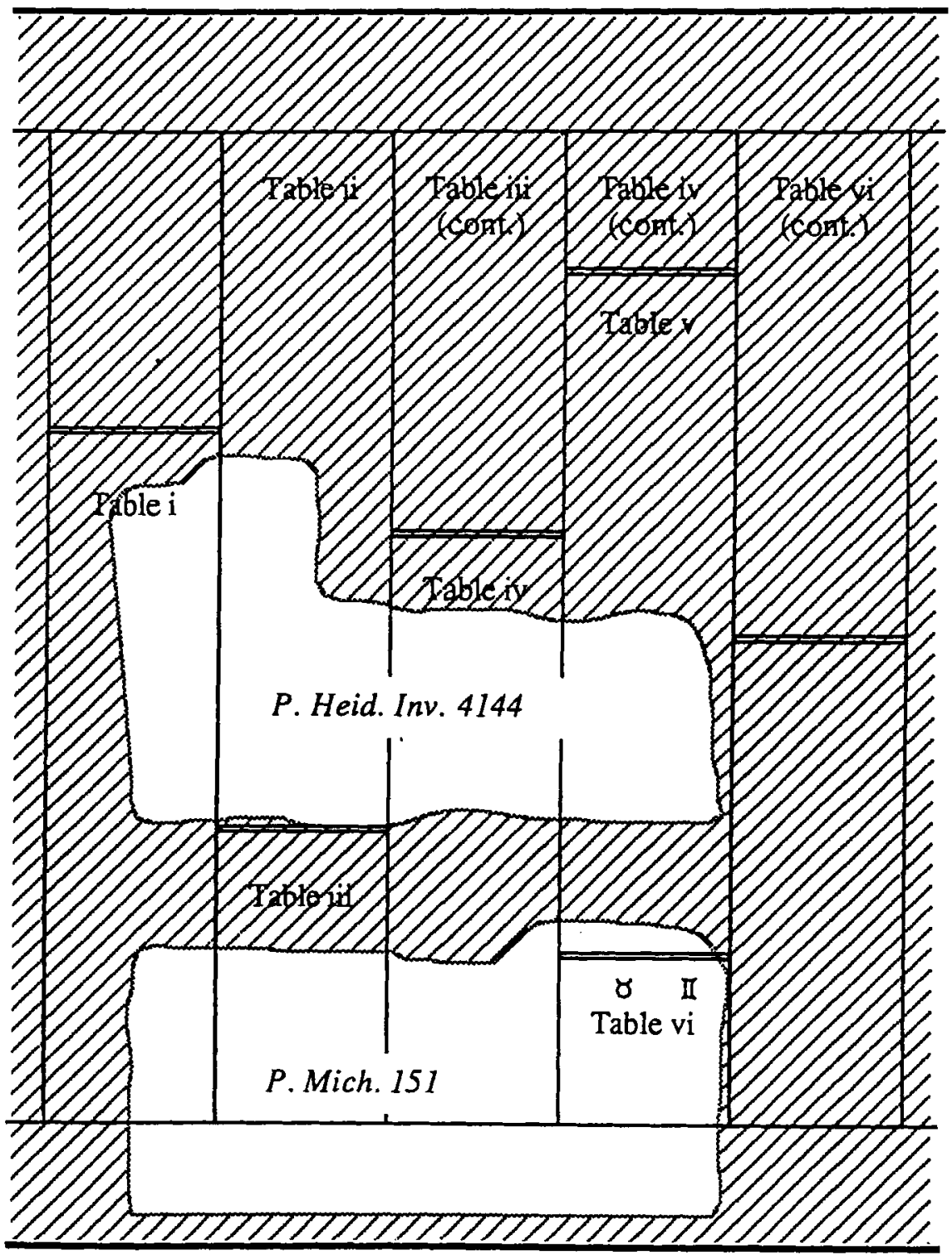

Figure 1. Position of the papyrus fragments in relation to the original document 
exactly without making any presuppositions about their significance. As a specimen, Table iv is shown in Fig. 2. In each table, the first column is a sequence of two or three digit sexagesimal numbers, while the second column is a list of index numbers, always beginning with $\frac{1}{3}$ and $\frac{2}{3}$, and then ascending by units from 1 through 30 . One quickly verifies that the first column consists simply of multiples of a base number by the indices in the second column; the products are truncated to two places for all indices greater than 2 . The base numbers (corresponding to index 1 ) and final numbers (corresponding to 30 ) are as follows:

\begin{tabular}{l|l|l} 
Table & base & final \\
\hline ii & $1 ; 54$ & 57 \\
iii & $1 ; 21,30$ & $40 ; 45$ \\
iv & $1 ; 32,18$ & $46 ; 9$ \\
v & $1 ; 48,27$ & $54 ; 13,30$ \\
vi & $1 ; 29,48$ & $44 ; 54$
\end{tabular}

Which digit represents units is, of course, not indicated in the papyrus. Some traces of the first column of Table $i$ are visible: the last numerals of the numbers corresponding to indices 24,26 , and 28 were, respectively, 4,6 , and 8 .

Each table presumably had a heading in the original document, but the heading for Table vi is alone preserved: TAYPOY $\triangle I \Delta-$ $Y M[\Omega N]$, i.e. "Of Taurus (and) Gemini". The only known context in ancient astronomy in which these two signs were treated as a unit is the System A Mars scheme, so that it is a reasonable supposition that each of the six tables was associated with one of the six System A zones. The base numbers do not suggest any obvious meaning in terms of Mars's motions, but the final numbers hover around the planet's mean synodic arc. As Neugebauer points out, the arithmetical mean of the five attested values is $48 ; 36,18$, which is close to the mean synodic arc $\bar{w}=48 ; 43,18,30^{\circ}$ assumed in System A. ${ }^{13}$ It is therefore already very probable that the final numbers of the six tables were the synodic arcs prescribed for the six zones in a modified form of the System A scheme.

This fact allows us to obtain an approximate value for the final number in Table i. ${ }^{14}$ The synodic arcs $w_{i}$ prescribed in a System A 


\begin{tabular}{rrr|rr|rrr}
0 & 30 & 46 & 0 & 20 & 23 & 4 & 15 \\
1 & 1 & 32 & 0 & 40 & 24 & 36 & 16 \\
1 & 32 & 18 & 1 & 26 & 9 & 17 \\
3 & 4 & 36 & 2 & 27 & 41 & 18 \\
4 & 36 & 3 & 29 & 13 & 19 \\
6 & 9 & 4 & 30 & 36 & 20 \\
7 & 41 & 5 & 32 & 18 & 21 \\
9 & 13 & 6 & 33 & 50 & 22 \\
10 & 46 & 7 & 35 & 22 & 23 \\
12 & 18 & 8 & 35 & 55 & 24 \\
13 & 50 & 9 & 38 & 27 & 25 \\
15 & 23 & 10 & 39 & 59 & 26 \\
16 & 55 & 11 & 41 & 32 & 27 \\
18 & 27 & 12 & 43 & 4 & 28 \\
19 & 59 & 13 & 44 & 36 & 30 \\
21 & 32 & 14 & 46 & 9 &
\end{tabular}

Figure 2. Transcription and restoration of table iv of the papyrus. (Numerals in bold face are legible on the fragment; incorrect digits are underlined.)

partition of the ecliptic into zones of length $\alpha_{i}$ must be related to the mean synodic arc $\bar{w}$ in order that the predicted longitudes will not progressively deviate from the true longitudes:

$$
\sum \frac{\alpha_{i}}{w_{i}}=\frac{360^{\circ}}{\bar{w}} .
$$

Since for Mars $\bar{w}=48 ; 43,18,30^{\circ}$, the equation

$$
\frac{60^{\circ}}{w_{i}}+\frac{60^{\circ}}{57^{\circ}}+\frac{60^{\circ}}{40 ; 45^{\circ}}+\frac{60^{\circ}}{46 ; 9^{\circ}}+\frac{60^{\circ}}{54 ; 13,30^{\circ}}+\frac{60^{\circ}}{44 ; 54^{\circ}}=\frac{360^{\circ}}{48 ; 43,18,30^{\circ}}
$$

leads to a value of $53 ; 31,33, \ldots$ for $w_{i}$. This assumes, of course, that 
the scheme of the papyrus conformed exactly to the System A period relation for Mars. It is implausible, at any rate, that $w_{i}$ would have been far outside the range between $53^{\circ}$ and $54^{\circ}$. In the light of the preserved traces of Table $\mathrm{i}$ in the papyrus, $w_{i}$ probably was exactly $53^{\circ}$.

Neugebauer made the natural assumption that the six tables in the papyrus pertained to the six zones of the ecliptic in zodiacal order. Thus, knowing that Table vi belonged to Taurus and Gemini, he obtained the following correspondences:

$$
\begin{array}{ll}
\text { zone } & w_{i} \\
1(\varangle+\mathbb{I}) & 44 ; 54 \\
2(\Xi+\Omega) & 53 \\
3(\pi+\simeq) & 57 \\
4(m+\neg) & 40 ; 45 \\
5(\sigma+=) & 46 ; 9 \\
6(x+r) & 54 ; 13,30
\end{array}
$$

Unfortunately, this pattern is astronomically absurd. It would have Mars's synodic arcs increasing from a minimum to a peak value over each half of the ecliptic, with abrupt drops after each half. In fact the synodic arcs of any planet except Mercury vary from a single minimum near apogee to a single maximum near perigee, and for Mars this variation is so pronounced that it could hardly be mistaken.

If we allow that Tables $i$ through vi were not in zodiacal order, then only two sequences exist that would give Mars a single minimum synodic arc in the vicinity of its apogee in Cancer, and a single maximum near the perigee in Capricorn:

\begin{tabular}{lll} 
zone & \multicolumn{2}{c}{$w_{i}$} \\
& (a) & (b) \\
$1(\varangle+I)$ & $44 ; 54$ & $44 ; 54$ \\
$2(\sigma+\Omega)$ & $40 ; 45$ & $40 ; 45$ \\
$3(m+\Omega)$ & $46 ; 9$ & $46 ; 9$ \\
$4(\pi+\curvearrowright)$ & $54 ; 13,30$ & 53 \\
$5(\sigma+=)$ & 57 & 57 \\
$6(x+r)$ & 53 & $54 ; 13,30$
\end{tabular}


These differ only in the assignment of zones 4 and 6. Sequence (a) has in its favour that it preserves the zodiacal order expected in the papyrus, except for the (accidental?) exchange of zones 1 and 5. But sequence (a) also exhibits a sinusoidal variation of such striking symmetry that it can hardly be doubted that this was the scheme of the papyrus. The horizontal bars in Fig. 3 show the prescribed $w$ values plotted as a function of the starting longitude. If we hypothesize that these $w$ 's were actually computed as some function of longitude evaluated at a particular point in each zone (say the middle or the beginning of each zone), the function will have looked roughly like the curved line plotted in Fig. $3 .^{15}$ The less orderly graph of the Babylonian System A w's is similarly displayed in Fig. 4. As we have seen, the Babylonian numbers were derived from tallies of observed planetary phases over whole zones, not by a method equivalent to evaluating some continuous function at $60^{\circ}$ intervals; hence the curve in Fig. 4 is based on a false hypothesis,

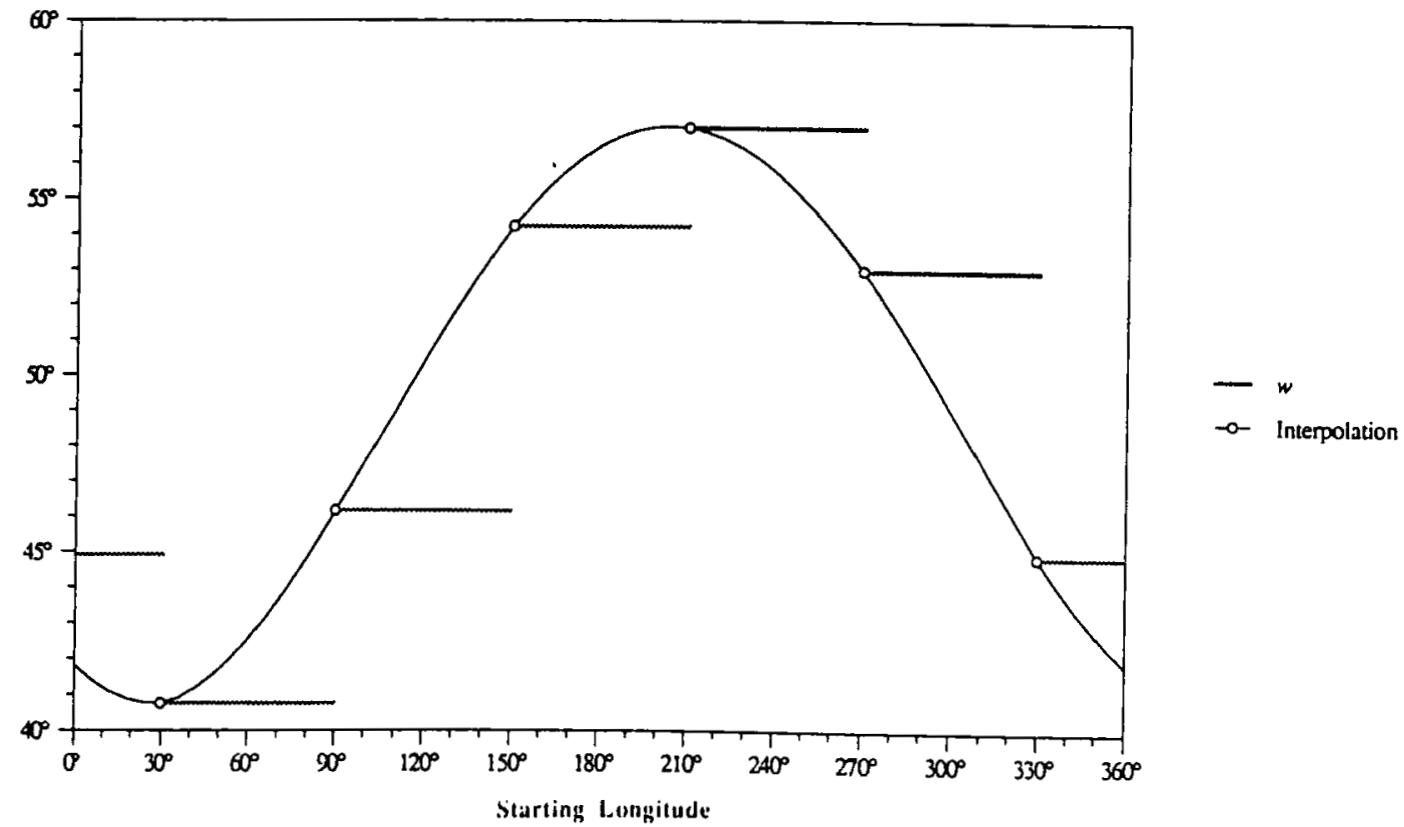

Figure 3. P. Heid Inv. $4144+P$. Mich. 151 
and its asymmetry is to be expected. But the much more regularly arranged $w$ 's of the papyrus do look as if they were found as values derived by some sort of theory for specific longitudes. This is borne out by the dismal performance of the papyrus scheme in predicting longitudes of Mars's phases. Fig. 5 shows the synodic arcs predicted by both schemes (taking account of zone transitions) as a func ion of starting longitude, juxtaposed with an estimate of the 'true function' of synodic arc between consecutive first stations interpolated from a series of synodic arcs computed according to modern theory. ${ }^{16}$ This shows how good an approximation the Babylonian scheme is; but meanwhile the synodic arcs predicted by the papyrus can err by more than $20^{\circ}$, and the accumulated error in three consecutive synodic periods can exceed $30^{\circ}$ !

There are three reasons why the w's of the papyrus could not have been derived from the same kind of observational data as their System A counterparts. First, it is highly unlikely that the

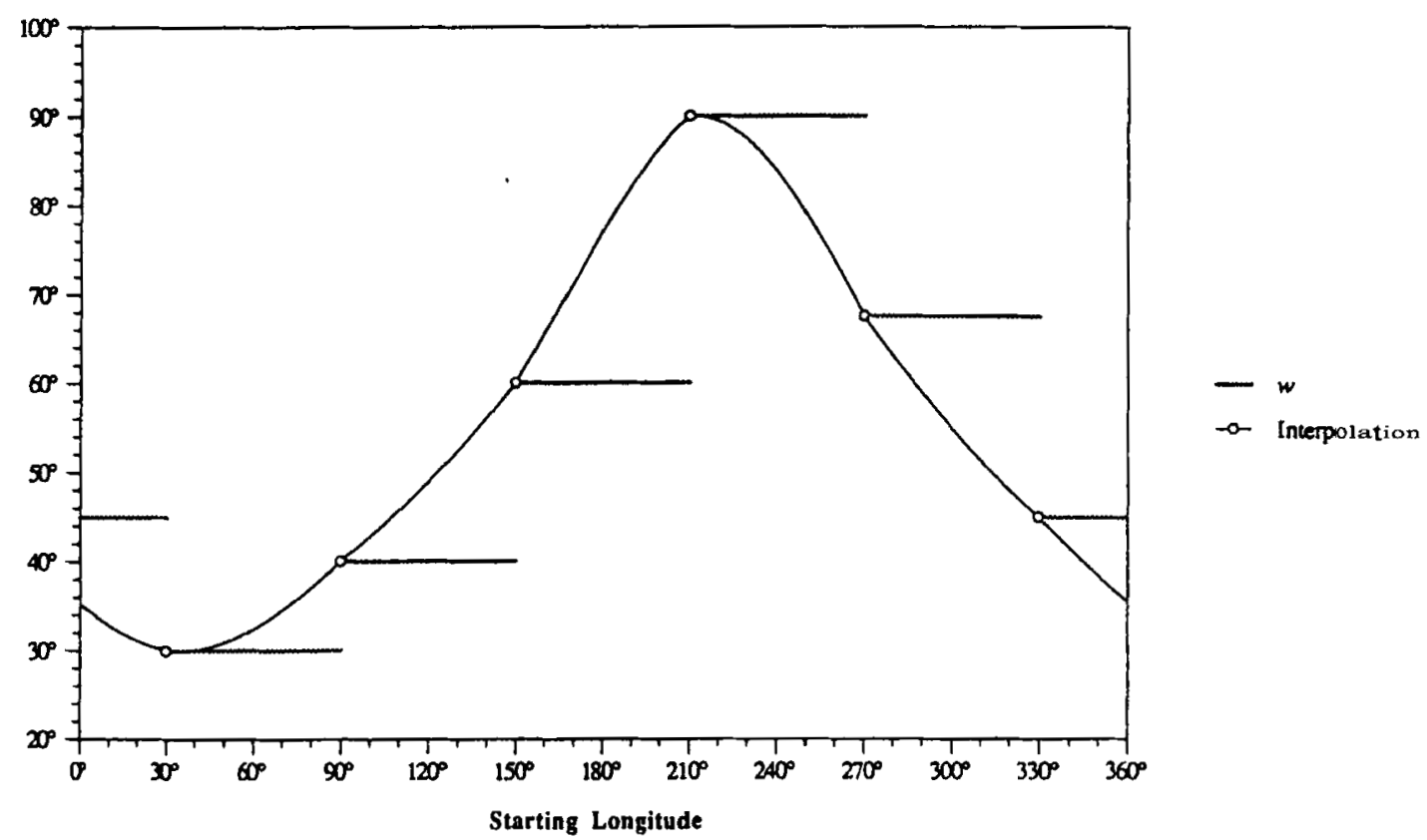

Figure 4. Babylonian System A 
transmission of Babylonian predictive methods into Greek, extensive though it was, included details of how the methods were empirically derived (what we know of this is purely modern deduction). Secondly, even with this knowledge, a Greek astronomer would have lacked the extensive observational records necessary to make the tallies. And thirdly, even if a Greek astronomer had possessed such a record, and had undertaken to check the accuracy of the Babylonian tallies, he should have arrived at a distribution of phases little different from the System A pattern. Even if we suppose that this imaginary astronomer used very bad observations, variations in frequency of phases among the six zones as slight as those predicted by the papyrus scheme could never have been derived from observations extending over an interval of less than several centuries.

In short, the papyrus scheme prescribes for Mars a pattern of motion of great regularity, but that considerably underestimates

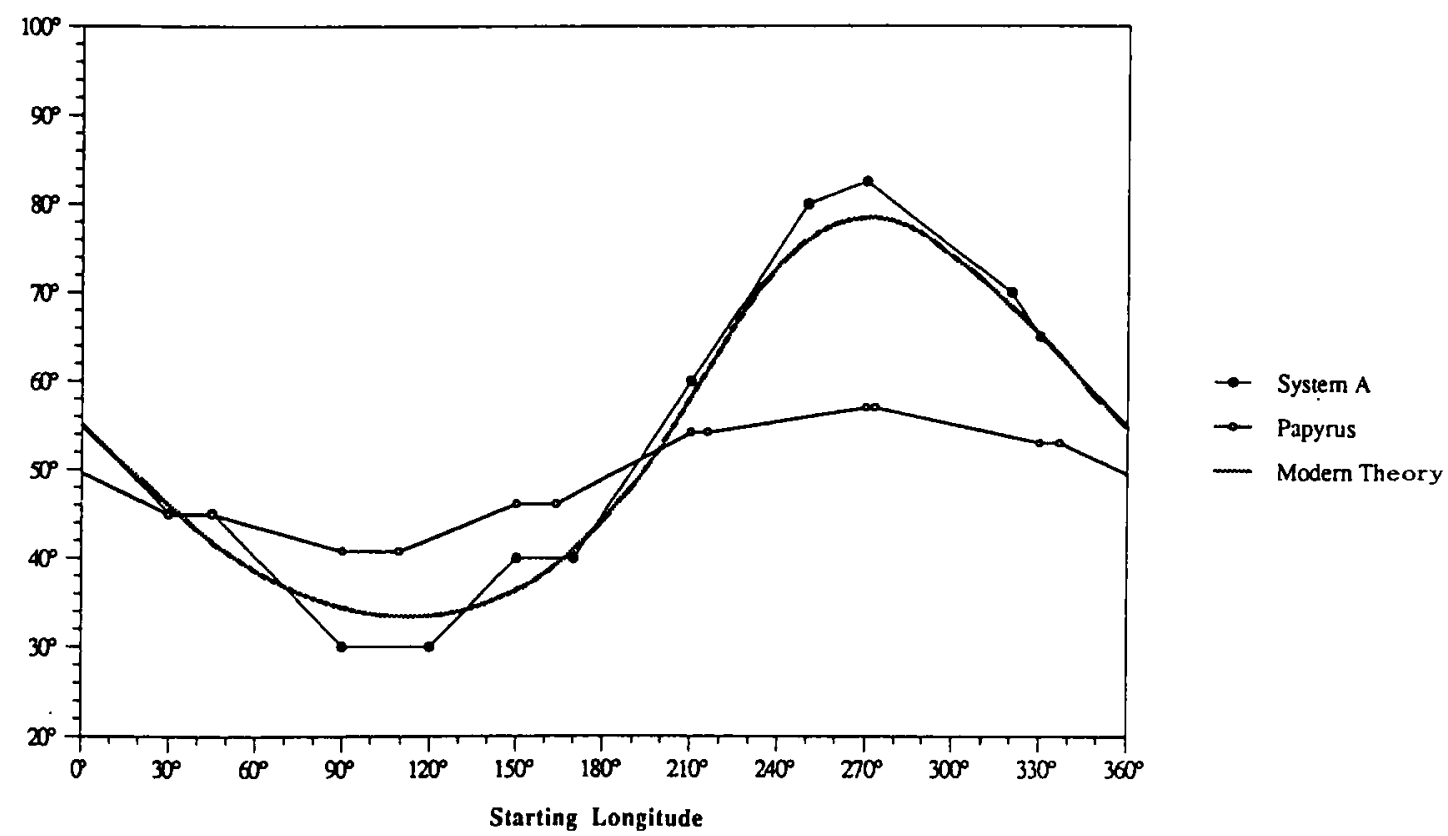

Figure 5. Synodic Arcs between First Stations 
the variation in the planet's synodic arcs. We have seen that the w's are not of Babylonian ancestry; but the only plausible source remaining for them is a geometrical model. Although I do not expect that an exact reconstruction of the method by which the attested numbers were derived is likely to be found, the following procedure shows how considerations easily within the grasp of a Greek astronomer between Hipparchus and Ptolemy lead to synodic arcs very like those in the papyrus.

We begin by giving Mars an epicycle-eccentre model, that is, the planet revolves uniformly around an epicycle that revolves around a deferent circle not concentric with the earth. In this kind of planetary model, the epicycle causes the synodic anomaly of the planet, while the eccentric deferent is responsible for the zodiacal anomaly. We may be supposed to have values for the epicycle radius $r$ and the eccentricity $e$ (in terms of the deferent's radius), the longitude $\lambda_{A}$ of the deferent's apogee, and the periods of the two revolutions. We want to determine the synodic arc between two successive occurrences of some phase of Mars predicted by this model when the longitude of Mars at the first phase is $30^{\circ}, 90^{\circ}$, $150^{\circ}, 210^{\circ}, 270^{\circ}$, and $330^{\circ}$, i.e. at the beginning of each $60^{\circ}$ zone. ${ }^{17}$ It will be convenient to restrict our attention to oppositions or conjunctions of Mars with the sun, because at these phases the planet's longitude is identical to the longitude of the epicycle's centre $C$; in other words we can forget the epicycle at these phases and treat Mars as if it were a planet travelling on a simple eccentre. ${ }^{18}$ The problem can now be expressed more generally: given that at some moment $C$ is at longitude $\lambda_{1}$ and the mean sun at longitude $\Lambda_{1}$, we have to find the next moment when the difference between the longitudes of $C$ and the mean sun, $\lambda_{2}-\Lambda_{2}$, equals $\lambda_{1}-\Lambda_{1}$.

A direct trigonometrical solution of this problem is possible, though not easy; I am not convinced that an astronomer before Ptolemy would have worked in this way. ${ }^{19}$ The task could be made easier by tabulating the longitudes of $C$ and the mean sun at regular intervals (say, daily) for two zodiacal revolutions. The daily positions of the mean sun increase, of course, by constant differences. One can calculate the daily position of $C$ according to the correct trigonometric formula; but without much loss of accuracy we can even represent the daily increment in longitude of $C$ as a linear zigzag function 
oscillating with the period of revolution of $C$, and attaining its minimum and maximum respectively at the apogee and perigee. ${ }^{20}$ The mean of this function will be Mars's mean daily motion in longitude; either its maximum or minimum can be set to the maximum or minimum daily motion resulting from the assumed eccentricity $e$ (we are not free to choose both extremes because of the symmetry of the zigzag function with respect to its mean). The summation of our daily motion function will be the longitude of $C$. We also tabulate the difference between the longitudes of $C$ and the mean sun.

Now, we find the date on which the longitude of $C$ is at $30^{\circ}$, and note the corresponding solar elongation (interpolating if necessary). Then we look for the next date when the elongation is the same value. The longitude of $C$ at this date minus $30^{\circ}$ will be the synodic arc corresponding to a starting longitude of $30^{\circ}$, while the difference in time between the two dates gives the synodic period. We do the same for each zone. The resulting set of six synodic arcs may, however, be collectively too large or too small to be used as w's with the System A rules. The $w$ 's, as we have seen, must conform to the equation

$$
\sum \frac{60^{\circ}}{w_{i}}=\bar{w}
$$

This condition can be satisfied by multiplying our synodic arcs by a scaling factor.

I have tried this procedure using various assumed eccentricities and longitudes of the apogee (these are the only parameters for which we have much choice). Using a correct trigonometrical function for the equation of centre, I found synodic arcs in satisfactory agreement with the papyrus values when the assumed eccentricity was $\frac{1}{13}$ and the apogee at $s 20^{\circ}$ :

$\begin{array}{lcccccc}\text { starting longitude } & 30^{\circ} & 90^{\circ} & 150^{\circ} & 210^{\circ} & 270^{\circ} & 330^{\circ} \\ \text { synodic arc } & 44.86^{\circ} & 41.97^{\circ} & 45.38^{\circ} & 53.86^{\circ} & 57.71^{\circ} & 52.09^{\circ}\end{array}$

These imply a mean synodic arc of

$$
\frac{60^{\circ}}{44.86^{\circ}}+\frac{60^{\circ}}{41.97^{\circ}}+\frac{60^{\circ}}{45.38^{\circ}}+\frac{60^{\circ}}{53.86^{\circ}}+\frac{60^{\circ}}{57.71^{\circ}}+\frac{60^{\circ}}{52.09^{\circ}} \approx 48 ; 41, \ldots
$$


which is near enough to the desired value that no scaling is needed. For the approximation using a zigzag function, I obtained synodic arcs close to the papyrus values when the ratio between maximum and minimum daily motion was 11:9 (i.e. an eccentricity of about $\frac{1}{10}$ ), and the apogee was again at $320^{\circ}$. Before scaling, the synodic arcs found were:

starting longitude $\quad 30^{\circ} \quad 90^{\circ} \quad 150^{\circ} \quad 210^{\circ} \quad 270^{\circ} \quad 330^{\circ}$ $\begin{array}{lllllll}\text { synodic arc } & 46.78^{\circ} & 41.69^{\circ} & 47.66^{\circ} & 55.40^{\circ} & 60.04^{\circ} & 53.53^{\circ}\end{array}$

For these values, we find

$$
\frac{60^{\circ}}{46.78^{\circ}}+\frac{60^{\circ}}{41.69^{\circ}}+\frac{60^{\circ}}{47.66^{\circ}}+\frac{60^{\circ}}{55.40^{\circ}}+\frac{60^{\circ}}{60.04^{\circ}}+\frac{60^{\circ}}{53.53^{\circ}} \approx 50 ; 7, \ldots
$$

Hence these synodic arcs have to be multiplied by about $\frac{48 ; 43}{50 ; 7}$ to make a viable zone scheme. The following table compares the $w$ 's found by the two foregoing reconstructions with the papyrus values; there is not much to choose between them.

$\begin{array}{llll}\text { zone } & \text { equation } & \text { zigzag } & \text { papyrus } \\ 1(\varangle+\pi) & 44 ; 51 & 45 ; 29 & 44 ; 54 \\ 2(\sigma+\Omega) & 41 ; 58 & 40 ; 32 & 40 ; 45 \\ 3(m+\Omega) & 45 ; 22 & 46 ; 20 & 46 ; 9 \\ 4(m+\Omega) & 53 ; 51 & 53 ; 52 & 54 ; 13,30 \\ 5(z+=) & 57 ; 42 & 58 ; 22 & 57 \\ 6(x+r) & 52 ; 5 & 52 ; 3 & 53\end{array}$

The parameters assumed in these reconstructions are historically plausible. The assumed apogee, $\approx 20^{\circ}$, may be compared to Ptolemy's $\approx 25 ; 10^{\circ}$. The eccentricity of Mars's deferent that one should expect to find for an epicycle-and-eccentre model without equant depends (as Ptolemy points out in Almagest X 6) on the kind of observations used. From observations of solar oppositions he derives an eccentricity of about $\frac{1}{5}$; but to get the right amount of variation in the length of Mars's retrograde arcs one needs a much 
smaller eccentricity, ideally about $\frac{1}{10}$. The eccentricity in the model for Mars that lies behind the papyrus scheme was apparently chosen to accommodate the variation of the retrograde arcs, and not observed oppositions. An assumed eccentricity of $\frac{1}{5}$ would have resulted in much better predictions of synodic arcs - not very different, in fact, from the Babylonian System A w's.

This takes care of the chief problem left unsolved by Neugebauer, namely the correct sequence and theoretical derivation of the $w$ 's in the papyrus. A minor puzzle remains: what was the purpose of the multiplication tables in the papyrus? I think that a clue to the answer was already indicated by Neugebauer, when he pointed out that the $w$ 's in the papyrus do not led to convenient terminating sexagesimal fractions as transition coefficients, such as one generally finds in Babylonian System A schemes. ${ }^{21}$ This observation remains valid no matter how the zones are reordered. Consequently whoever invented the papyrus scheme cannot have intended that the synodic arcs crossing zone boundaries be calculated in the Babylonian way. I suggest that when addition of $w_{i}$ would have led to a boundary crossing, one was expected to look in the first column of the appropriate multiplication table for the number closest to the degrees in the zone $i$ between the starting longitude and the boundary, and subtract the corresponding index in the second column from 30. One then looked up the remainder in the second column of the table for zone $i+1$, and read off the corresponding number in the first column as the number of degrees to be added after the zone boundary. The number 30 was probably chosen merely to produce tables of a reasonable length, while the two fractional indices at the beginning of each table would have been used for rough interpolation.

Other details of the scheme are beyond recovery. For example, we do not know which phases of Mars were to be computed directly by the six-zone pattern. The derivation of the synodic arcs from an epicycle-eccentre model, if carried out in the way that I have suggested, would be strictly applicable only to conjunctions and oppositions. But a Greek astronomer may well have assumed that the same arcs subsist between recurrences of other phases. This would amount to van der Waerden's "sun-distance principle", which is already implicit in the Babylonian scheme: the principle states 
that a particular phase of a planet always takes place at the same elongation from the mean sun ${ }^{22}$. There must have been a rule for finding the dates of phases, which may have been an adaptation of the System A linear relation between synodic arc and synodic period. And one would expect, in a Greco-Roman context where astrological applications were important, to find rules for interpolating between phases to find daily positions, or at least the dates when the planet entered each zodiacal sign.

In conclusion, two general remarks may be made about our papyrus that are suggestive of the character of Greek astronomy before Ptolemy (for although the papyrus apparently dates from the third century, the scheme to which it belonged betrays no influence of Ptolemy's innovations). First, it illustrates a prominent aspect of the relationship between Babylonian "arithmetical" and Greek "geometrical" astronomy in this period. Numerical parameters are being determined for geometrical cinematic models of the planetary motions, but when it comes to applying the new theory to predicting longitudes, the structure of the Babylonian schemes is preserved, albeit with new numbers derived from the geometrical model. Tables that, like Ptolemy's, were expressly designed to 'exhibit the uniform circular motion' in the cinematic models, did exist before the Almagest (as we know from Indian astronomy), but there is no trace of these precursors in the known papyri. Secondly, the papyrus scheme, like the model of Mars's motion on which it depends, predicts longitudes of the planet's phases that are so inaccurate that it is impossible to believe that they were ever confronted with even the crudest observations. The provincial Egyptian astrologers who were responsible for most of the preserved astronomical papyri should perhaps not be expected to have looked too closely into the quality of their tables. ${ }^{23}$ But what is one to say for the astronomer who devised the scheme in the first place? Documents like our papyrus argue forcefully against the supposition that the sophisticated methodology of Ptolemy's Almagest was at all typical of the astronomy of his time. 


\section{BIBLIOGRAPHY}

Aaboe, A.

1958: "On Babylonian Planetary Theories", Centaurus 5, pp. 209-77.

1980: "Observation and Theory in Babylonian Astronomy", Centaurus 24, pp. 14-35.

Houlden, M. A., and Stephenson, F. R.

1986: A Supplement to the Tuckerman Tables, Philadelphia.

Jones, $A$.

1983: "The Development and Transmission of 248-Day Schemes for Lunar Motion in Ancient Astronomy", Archive for History of Exact Sciences 29, pp. 1-36.

1991: "Models and Tables in Ancient Astronomy, 200 B.C. to A.D. 300", Aufstieg und Niedergang der Römischen Welt II 37.4 (forthcoming).

\section{Kugler, F. X.}

1900: Die Babylonische Mondrechnung, Freiburg i. B..

Neugebauer, 0 .

1942: "On some Astronomical Papyri and Related Problems of Ancient Geography", Transactions of the American Philosophical Society N.S. 32, pp. 251-63.

1960: "A New Greek Astronomical Table (P. Heid. Inv. 4144+P. Mich. 151)", Hist. Filos. Medd. Dan. Vid. Selsk. 39.1

1967: "Problems and Methods in Babylonian Mathematical Astronomy", Astronomical Journal 72, pp. 964-72.

1975: A History of Ancient Mathematical Astronomy, Berlin, etc.

1988: "A Babylonian Lunar Ephemeris from Roman Egypt", A Scientific Humanist: Studies in Memory of Abraham Sachs, ed. E. Leichty, M. de J. Ellis, and P. Gerardi. (Occasional Publications of the Samuel Noah Kramer Fund, 9) Philadelphia, pp. 301-304.

Neugebauer, O., and Parker R.

1969: Egyptian Astronomical Texts, Vol. 3. Providence.

Sachs, A. J., and Hunger $\mathrm{H}$.

1988: Astronomical Diaries and Related Texts from Babylonia. Vol. I: Diaries from 652 B.C. to 262 B.C. (Österreichische Akademie der Wissenschaften, phil.-hist. K1., Denkschriften 195), Vienna.

van der Waerden, B. L.

1947: "Egyptian 'Eternal Tables"', Koninklijke Nederlandsche Akademie van Wetenschappen. Proceedings 50, pp. 536-47 and 782-88.

1960: "Babylonische Methoden in ägyptischen Planetentafeln". Vierteljahrsschrift der Naturforschenden Gesellschaft in Zürich 105, pp. 97-144.

1972: “Aegyptische Planetenrechnung", Centaurus 16, pp. 65-91.

1974: Science Awakening II: The Birth of Astronomy, Leyden.

\section{REFERENCES}

1. Neugebauer, 1960 , repudiating an incorrect interpretation of $P$. Mich. 151 that he had earlier given in Neugebauer, 1942. P. Mich. 151 was originally published in Michigan Papyri v. 3 (Ann Arbor: 1936) p. 118. 
2. Neugebauer, 1975 , pp. $946-48$.

3. The seminal work was Kugler, 1900.

4. See for example Neugebauer, 1967, p. 972, and Neugebauer, 1975, pp. 589-90 and 604 .

5. Van der Waerden, 1947, 1960, and 1972.

6. Neugebauer and Parker, 1969, pp. 235-40, and Neugebauer, 1975, pp. 607 and 789-90.

7. Evidence does exist for Greek knowledge and adaptation of Babylonian schemes for the other planets. Moreover, in the light of the astonishing papyrus recently published by Neugebauer, 1988, it is now evident that even the much more complex Babylonian lunar schemes were known in Roman Egypt. For a fuller discussion of the nature and extent of the transmission of the Babylonian schemes, see Jones, 1991, part. 3.

8. For details, see Neugebauer, 1975, pp. 454-460.

9. Aaboe, 1980. Conveniently, Aaboe uses Mars for his illustration of the procedure.

10. The large corpus of surviving Diaries is in the course of publication: Sachs \& Hunger, 1988. On the Goal-Year Periods see Neugebauer, 1975, pp. 554-55. This set of period relations is well attested in the Seleucid period, but similar relations were certainly known long before. See van der Waerden, 1974, pp. 107-108.

11. Aaboe, 1958, pp. 245-251. Cf. Figure 5 below.

12. Adapted from Fig. 1 in Neugebauer, 1960, p. 4. See this article for further papyrological description and a photograph.

13. Neugebauer, 1975, p. 947.

14. Here Neugebauer incorrectly takes the number that would make the arithmetic mean of the six arcs equal the Babylonian mean value.

15. Each pair of points in the graph is joined by the fifth-degree polynomial passing through the preceding and following pairs of points.

16. I derived these longitudes from Houlden \& Stephenson, 1986. For a comparison of System A with first and last visibilities computed according to modern theory, see Aaboe, 1958, pp. 249-50.

17. The beginning of the zone is arguably a better choice than the middle, because it avoids zone transitions.

18. This theorem is proved by Ptolemy, Almagest X 6, and I can see no reason why it cannot have been known earlier.

19. See Aaboe, 1958, for the procedure.

20. A possible model for this representation is furnished by the zigzag functions widely used in Hellenistic astronomy for lunar daily motion (Jones, 1983).

21. Neugebauer, 1975, p. 948.

22. Van der Waerden, 1974, pp. 266-68.

23. There are many incorrect digits in the preserved fragments of our papyrus (cf. Neugebauer, 1960, p. 7), where a check was possible by simple addition. 